\title{
Pressure/volume curves and lung computed tomography in acute respiratory distress syndrome
}

\author{
J-J. Rouby*, Q. Lu*, S. Vieira\#
}

Pressurelvolume curves and lung computed tomography in acute respiratory distress syndrome. J-J. Rouby, Q. Lu, S. Vieira. (C) ERS Journals Ltd 2003.

ABSTRACT: Pressure/volume $(P I V)$ curves can be measured by static methods, constant or sinusoidal flow methods and the dynostatic method that allows a breath-tobreath determination of $P I V$ curves. Recent ventilators are equipped with specific flow generators and software aimed at obtaining $P / V$ curves without disconnecting the patient from the ventilator.

The most recent generation of computed tomography scanners allows the quantitative determination of lung aeration, lung volumes (gas and tissue), alveolar recruitment and lung overinflation of the whole lung. In the supine position, the acute respiratory distress syndrome (ARDS) lung is characterised by an increase in lung tissue that predominates in upper lobes and a massive loss of aeration that predominates in lower lobes.

In a minority of ARDS patients, the loss of aeration is homogeneously distributed. The overall lung volume of upper lobes is preserved suggesting an alveolar floodinginduced loss of aeration. In contrast, the overall lung volume of lower lobes is reduced because the heart and the abdomen exert an external compression that contributes to the loss of aeration. The $P I V$ curve is a lung recruitment curve and the chord compliance indicates the potential for recruitment. In such patients, alveolar recruitment resulting from positive end-expiratory pressure is not accompanied by lung overinflation.

In a majority of acute respiratory distress syndrome patients, upper lobes remain partially or totally aerated despite a marked regional increase in lung tissue. The upper lobes' overall lung volume is either normal or increased, suggesting that the lung does not collapse under its own weight as generally believed. In lower lobes, the overall lung volume is reduced because the heart and the abdomen exert an external compression that contributes to the loss of aeration. The pressure/volume curve is influenced by the recruitment of poorly and nonaerated lung regions and by the mechanical properties of the part of the lung remaining aerated. In such patients, alveolar recruitment resulting from positive end-expiratory pressure $>10 \mathrm{cmH}_{2} \mathrm{O}$ is preceded and accompanied by lung overinflation.

Eur Respir J 2003; 22: Suppl. 42, 27s-36s.
*Réanimation Chirurgicale Pierre Viars, Dept of Anesthesiology, Hospital Pitié-Salpétrière, University Paris VI, Paris, France, ${ }^{\#}$ Dept of Internal Medicine, Faculty of Medicine Federal University from Rio Grande Do Sul, Intensive Care Unit, Hospital de Clinicas de Porto Alegre, Porto Alegre, Brazil.

Correspondence: J-J. Rouby

Réanimation Chirurgicale Pierre Viars

Hôpital Pitié-Salpétrière

83 boulevard de 1'hôpital

75013 Paris

France

Fax: 33142177326

E-mail: jjrouby.pitie@invivo.edu

Keywords: Acute respiratory distress syndrome alveolar recruitment computed tomography scan

lung morphology

pressure/volume curve
Respiratory pressure/volume $(P / V)$ curves can be easily obtained at the bedside in critically ill patients whose lungs are mechanically ventilated. Many ventilators are now equipped with flow generators and software that allow the obtention and the analysis of the $P / V$ curve without disconnecting the patient from mechanical ventilation. Hypothesis and concepts serving for interpreting the $P / V$ curves of patients with acute respiratory distress syndrome (ARDS) have been deeply modified during the past $15 \mathrm{yrs}$. Initially believed to reflect the mechanical properties of the "baby" lung [1], the $P / V$ curve is now rather considered as a lung recruitment curve [2] which is markedly influenced by the regional distribution of the loss of lung aeration [3]. Considered in the past as a clinical tool for directly optimising ventilatory settings in patients with ARDS [4], the $P / V$ curve has today only an indirect impact on the ventilatory strategy because its interpretation at the bedside appears more complex than previously thought [5]. The aim of the present review is to describe the different methods for obtaining $P / V$ curves at the bedside and provide a rationale for their interpretation. The computed tomography (CT) analysis is also reviewed as well as differences in lung morphology characterising patients with ARDS.
Methods for measuring pressure/volume curves in ventilated patients

\section{Static methods}

The super-syringe method consists of inflation of the lungs in steps of $50 \mathrm{~mL}$ up to $2 \mathrm{~L}$ starting from the end-expiratory lung volume [1,4]. The paralysed and sedated patient is disconnected from the ventilator for a few seconds to empty the lungs completely. A 2-L syringe filled with pure oxygen is connected to the endotracheal tube and the inflation manoeuvre is started, the interval between two successive inflations being of $3 \mathrm{~s}$. The same manoeuvre can be performed during deflation, the entire procedure lasting about $60 \mathrm{~s}$. Airway pressure at the $\mathrm{Y}$ piece and lung volume changes resulting from the displacement of the piston are recorded simultaneously allowing the construction of the $P / V$ curve. 
The method has several disadvantages: the disconnection from the ventilator provokes a massive lung derecruitment in patients with acute lung injury, the obtention of $P / V$ curves in positive end-expiratory pressure (PEEP) conditions is difficult and a special piece of equipment that cannot be included in the ventilator is required. In addition, discrepancies can be evidenced between gas volume changes in the syringe and the resulting increase in lung volumes measured by indirect spirometry $[6,7]$. When changes in lung volume are measured, using inductance plethysmography, the $P / V$ curves exhibit a lesser degree of hysteresis (difference between the lung volumes during inflation and deflation at the same level of airway pressure) and the respiratory compliance during deflation is higher. These differences are observed only if the procedure is longer than $45 \mathrm{~s}$ and result from the gas exchange occurring in the lung during the manoeuvre: the oxygen uptake is only partially compensated for by the carbon dioxide production and changes in lung volume are slightly smaller than those that should have resulted from the displacement of the piston in the syringe. Administration of unwarmed and unhumidified gas also causes a displacement of the curve to the left $[7,8]$. Because of these methodological limitations, the super-syringe method is no longer in use at the bedside.

The inspiratory occlusion technique consists of measurement of plateau pressures corresponding to different tidal volumes following an end-inspiratory occlusion [9]. This technique is performed using a mechanical ventilator equipped with facilities for end-inspiratory and end-expiratory occlusions. It is not necessary to disconnect the patient from the ventilator and the loss of lung volume due to oxygen uptake is negligible. The patient has to be ventilated in a volumecontrolled mode with a constant inspiratory flow. The different tidal volumes are obtained by changing the respiratory rate while maintaining the inspiratory flow constant and are administered in a randomised sequence interrupted by periods of volume-controlled ventilation. Each occlusion manoeuvre is performed at end-inspiration and the plateau pressure is measured after a 3 -s occlusion. The $P / V$ curve is constructed by plotting the different plateau pressures with the corresponding tidal volumes and can be obtained at any PEEP level. This method has been extensively used to determine the lower and upper inflection points of the $P / V$ curve $[10,11]$ and quantify PEEP-induced alveolar recruitment in patients with ARDS $[12,13]$. The time required to perform the manoeuvre is around $15 \mathrm{~min}$, however, which renders the technique cumbersome in clinical practice.

\section{Quasistatic methods using a continuous inflation at constant, sinusoidal or decelerating flows}

An easy technique to obtain a $P / V$ curve without disconnecting the patient from the ventilator is to inflate the respiratory system by a constant flow issued from the ventilator $[14,15]$. This method is based on the fact that when the lungs are inflated at a constant inspiratory flow, the airway pressure change is inversely proportional to the compliance of the respiratory system $[16,17]$.

Constant flows $<10 \mathrm{~L} \cdot \mathrm{min}^{-1}$ allow the obtention of $P / V$ curves quasi-superimposable to those obtained with static methods [15, 18-20]. Such low flows can be delivered by most intensive care unit (ICU) ventilators in the absence of any special equipment. The ventilator has to be set in a volumecontrolled mode with a constant inspiratory flow, a tidal volume between $500-1500 \mathrm{~mL}$, an inspiration:expiration ratio of $80 \%$ and a respiratory frequency of 5 breaths $\cdot \mathrm{min}^{-1}$. With these particular ventilatory settings, a constant flow ranging between 3-9 $\mathrm{L} \cdot \mathrm{min}^{-1}$ is delivered over a period of $9.6 \mathrm{~s}$ and the $P / V$ curve can be displayed real-time on the screen of the ventilator. The measurement of the chord compliance (the slope of the linear part of the $P / V$ curve) and the determination of the upper and lower inflection points can be carried out using the mobile cursors available on the ventilator display screen. The entire procedure takes $1 \mathrm{~min}$ and is performed without disconnecting the paralysed patient from the ventilator $[18,19]$. Some recent ICU ventilators are equipped with specific flow generators that deliver constant flows between $1-10 \mathrm{~L} \cdot \mathrm{min}^{-1}$ allowing the obtention of static or quasi-static $P / V$ curves.

With constant flows $>10 \mathrm{~L} \cdot \mathrm{min}^{-1}$, the slope of the $P / V$ curve remains representative of the respiratory compliance whereas upper and lower inflection points are overestimated because of the resistive pressure generated by the high flow [14, 15]. A solution proposed to obviate the resistive factor is to subtract the resistive pressure generated in the respiratory tubings and the airways from the measured airway pressure [14, 21]. The resistive pressure generated by the tube connecting the $\mathrm{Y}$ piece to the proximal end of the endotracheal tube and by the endotracheal tube itself can be calculated as:

$$
P_{\text {res (tube) }}=\mathrm{K}_{1} \cdot \mathrm{V}+\mathrm{K}_{2} \cdot \mathrm{V}^{2}
$$

Where $\mathrm{V}$ is the inspiratory flow and $\mathrm{K}_{1}$ and $\mathrm{K}_{2}$ are coefficients determined in vitro for each type of connecting and endotracheal tube. The resistive pressure generated in the airways is calculated as the quotient between area of the pressure/ volume loop and area of the flow/volume loop when a constant flow is administered [14], or as the quotient between pressure and flow when an oscillating flow is used during the insufflation [21]. The dynamic low-flow inflation technique is an attractive alternative to the time-consuming multiple occlusion technique because it allows recording of $P / V$ curves during a single 6-s inflation following a prolonged expiration. However, it slightly underestimates chord compliance and the lower inflection point and requires a computer-controlled ventilator [21].

Recently, a new and attractive method has been proposed for measuring static $P / V$ curves during ordinary volume or pressure-controlled mechanical ventilation [22]. The "dynostatic" $P / V$ curve is obtained during a single tidal insufflation by measuring airway pressure at the distal tip of the endotracheal tube $[23,24]$ and calculating alveolar pressure from the dynamic tracheal pressure/volume loop. This breathto-breath determination of $P / V$ curves allows a continuous monitoring of respiratory mechanics without altering respiratory conditions by a nonphysiological manoeuvre [25]. When automatised, this continuous monitoring of "dynostatic" $P / V$ curves appears as a remarkable tool for detecting lung overinflation resulting from increases in PEEP and/or tidal volume [25].

\section{Measurement of chest wall and lung pressurelvolume curves}

It is well established that the shape of the $P / V$ curve of the respiratory system can be influenced by the chest wall elastic properties of patients with complicated abdominal surgery [26], large pleural effusions, enlarged heart, obesity and closed chest trauma. A lower inflection point $\leqslant 5 \mathrm{cmH}_{2} \mathrm{O}$ may be entirely explained by the chest wall [27]. As a consequence, it is of interest to briefly describe methods for measuring chest wall mechanics. The chest wall $P / V$ curve can be constructed by plotting lung volumes against oesophageal pressure, an estimation of pleural pressure. Oesophageal pressure can be measured by inserting into the mid-esophagus either a 
specifically designed catheter incorporating a thin-walled balloon inflated with air or, more simply, a water-filled Salem ${ }^{\mathrm{TM}}$ double-lumen catheter, commonly placed in the stomach of critically ill patients on mechanical ventilation [28]. The patient is kept in the half-sitting position in order to minimise the effect of weight of the mediastinum in the supine position and the water-filled Salem ${ }^{\mathrm{TM}}$ stomach tube is withdrawn into the oesophagus until the oesophageal pressure curve shows maximal respiratory-related fluctuations and minimal cardiac artifacts. Before measurement, an "occlusion test" consisting of a series of 3-5 spontaneous occluded inspiratory efforts is recommended [29]. A ratio between oesophageal pressure changes and occluded inspiratory pressure changes close to 1 indicates that the catheter is properly positioned and that oesophageal pressure is an acceptable estimation of pleural pressure. The lung $P / V$ curve is constructed by plotting lung volumes against transpulmonary pressures (differences between airway and oesophageal pressures). Obtention of chest wall $P / V$ curves using the "dynostatic" method and the Salem ${ }^{\text {TM }}$ gastric tube appears easy to perform and of clinical interest in patients with abdominal distension, cardiac enlargement and morbid obesity.

\section{Lung computed tomography: the quantitative analysis}

\section{Principles of image formation and quantitative assessment of lung aeration}

During CT, the X-rays hitting the CT detector depend on tissue absorption which is linearly correlated to physical tissue density [30]. X-ray attenuation is measured on each voxel, the elementary unit of volume which results from the product of the CT section thickness by the pixel area which, itself, depends on the matrix size. The spatial resolution of CT images is highly dependent on the size of the voxel which is $\sim 0.2 \mathrm{~mm}^{3}$ with the last generation of CT scanners. Overall lung volume (gas+tissue) is computed as the number of voxels present in a given lung region. Because the lung parenchyma is composed of gas and tissue with a physical density which is close to water density, it is possible to compute for any pulmonary region, the volume of gas, the volume of tissue and the volumic distribution of lung aeration [31, 32]. The CT attenuation of a given lung region is equivalent to its aeration: -300 Hounsfield Units (HU) indicates that the lung region is composed of $70 \%$ of tissue and $30 \%$ of gas (lung aeration= $30 \%) ;-750 \mathrm{HU}$ indicates that the lung region is composed of $75 \%$ of gas and $25 \%$ of tissue (lung aeration $=75 \%$ ). Classically, lung aeration is quantified in 4 categories: 1) normal aeration, defined by $\mathrm{CT}$ attenuations ranging between -900--500 HU (aeration ranging between $90 \%$ and $50 \%$ ), 2) overinflation, defined by CT attenuations <-900 HU (aeration $>90 \%$ ), 3) insufficient aeration, defined by CT attenuations ranging between -100-500 HU (aeration ranging between $10 \%$ and $50 \%$, 4) nonaeration, defined by $\mathrm{CT}$ attenuations $>-100 \mathrm{HU}$ (aeration $<10 \%)$.

\section{Lung aeration in normal and acutely injured lungs}

After the manual delineation of the lung parenchyma which remains a mandatory step of the quantitative analysis, lung volumes and pulmonary aeration can be measured using softwares based on the physical principles described above.

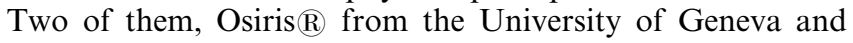

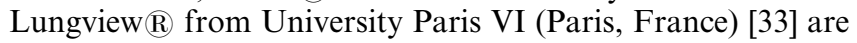
already available. Of particular interest for the clinician is the existence of a colour encoding system that attributes a specific colour to overinflated, normally, poorly and nonaerated lung areas according to their CT attenuations [34]. At endexpiration, the normal lung aeration ranges between $50 \%$ and $90 \%$. A lung aeration $>90 \%$ or $<10 \%$ is observed in $<1 \%$ of the normal lung parenchyma and only $7 \%$ of lung regions have an aeration ranging between $10-50 \%$ [32, 35]. Lung tissue volume is $\sim 1 \mathrm{~L}$, representing $30 \%$ of the end-expiratory lung volume and is equally distributed between right and left upper and lower lobes [32]. Functional residual capacity is around $2 \mathrm{~L}$, representing $70 \%$ of the overall lung volume and is smaller in the left lung than in the right lung due to the presence of the heart in the left hemithorax. In addition, functional residual capacity is slightly smaller in lower lobes than in upper lobes.

An increase in lung tissue is a characteristic hallmark of the ARDS lung. Pulmonary oedema, increased extravascular lung water, lung infection and lung inflammation contribute to the increase in lung tissue. A massive loss of aeration is also a prominent feature of the acutely injured lung. When the loss of aeration is isolated, without excess in lung tissue, it reflects atelectasis resulting from the mechanical compression or obstruction of distal bronchioles [36]. When the loss of aeration is associated with an increase in lung tissue, it likely reflects "alveolar flooding" resulting from the replacement of alveolar gas by oedema and/or inflammation. An excess of lung tissue can be detected in a given patient only if the amount of lung tissue normally present in the corresponding lung region of healthy humans is known [37].

\section{Measurement of alveolar recruitment and lung overinflation}

Alveolar recruitment resulting from the application of a positive pressure to the respiratory system (PEEP, sigh, recruitment manoeuvre) can be measured as the re-aeration of nonaerated lung regions according to the opening and collapse hypothesis $[1,36,38,39]$ or as the re-aeration of poorly and nonaerated lung regions if the hypothesis of alveolar flooding is taken into consideration [34]. Of peculiar importance is the requirement for assessing alveolar recruitment on the whole lung. It has been clearly shown that assessing alveolar recruitment on 1 or $3 \mathrm{CT}$ sections may result in a gross overestimation or underestimation of alveolar recruitment of the whole lung [40]. As reported in supine patients with ARDS, alveolar recruitment resulting from PEEP predominates in nondependent and cephalic lung regions, is rather limited in the diaphragmatic region and can even be negative (alveolar derecruitment) caudally to the diaphragmatic cupola [36, 41]. As a consequence, assessing alveolar recruitment on a single juxta-diaphragmatic CT section often underestimates recruitment of the whole lung. In contrast, because apical and juxta-hilar CT sections are more representative of upper lobes than of lower lobes, assessing alveolar recruitment on $3 \mathrm{CT}$ sections often overestimates recruitment of the whole lung [40].

Pulmonary overinflation can be defined as an excess of alveolar gas as compared to lung tissue. Very likely, a lung aeration $>90 \%$ (corresponding to CT attenuations $\leqslant-900 \mathrm{HU}$ ) is the threshold separating inflation from overinflation [35]. Lung emphysema complicating chronic obstructive pulmonary disease and characterised by lung overinflation and vascular destruction is also characterised by CT attenuations $<-900 \mathrm{HU}[42,43]$. Pulmonary overinflation resulting from mechanical ventilation predominates in caudal and nondependent lung regions [36]. As a consequence, assessing lung overinflation in patients with ARDS requires the analysis of 
the whole lung including CT sections caudal to the diaphragmatic cupola.

\section{Lung morphology in acute respiratory distress syndrome}

The different lung morphology patterns observed in patients with acute respiratory distress syndrome

ARDS is caused by a diffuse injury to the alveolar-capillary membrane that produces a high-permeability type pulmonary oedema. The resulting increase in lung tissue detected on lung CT [32] is distributed from the apex to the diaphragmatic cupola, predominant in upper lobes and frequently associated with a massive loss of aeration [36]. However, although the regional loss of aeration is always massive, the excess of lung tissue is absent or minimum in one-third of lower lobes [32]. Inversely, although the excess of lung tissue is constantly observed in cephalic parts of the lung, the aeration remains either partially preserved or entirely normal in two-third of upper lobes. One of the most intriguing results issued from CT studies of the whole lung is that the excess of lung tissue is not automatically associated with a significant loss of aeration.

In fact, the degree of aeration of upper lobes determines the lung morphology and the radiological pattern. In one fourth of patients with ARDS, the loss of aeration is massive and equally distributed within the lung parenchyma (fig. 1). In

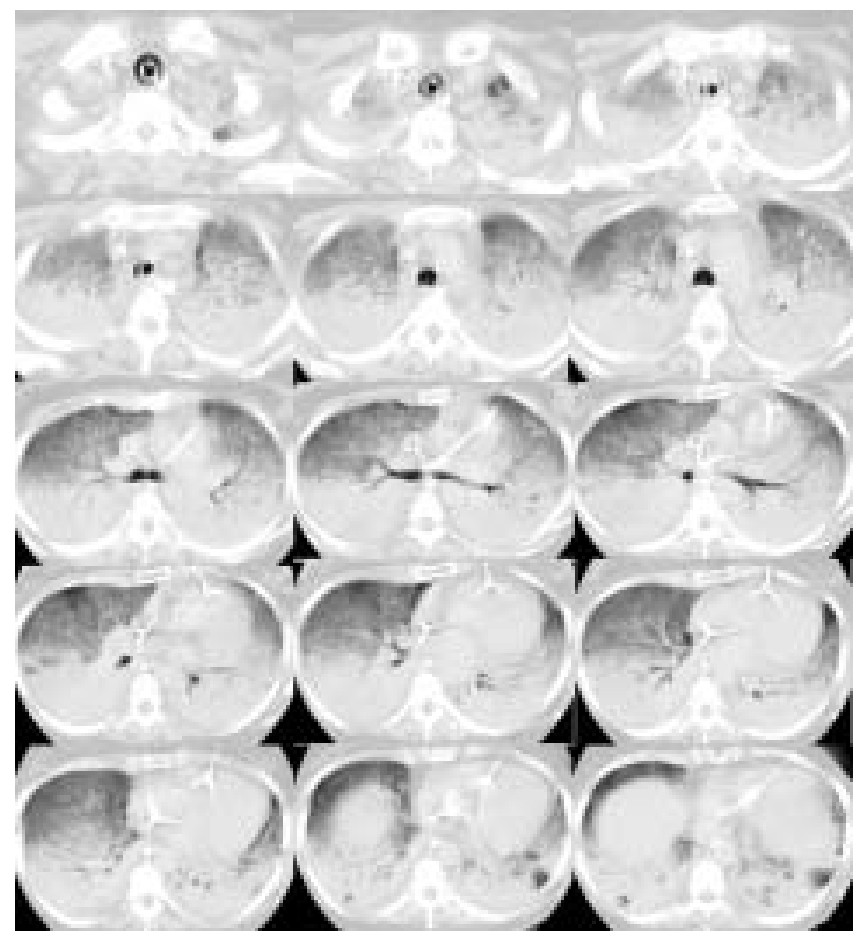

Fig. 1.-Computed tomography (CT) scan obtained from a 46-yr-old with "diffuse" acute respiratory distress syndrome caused by aspiration. Contiguous $1-\mathrm{cm}$ thick CT sections were obtained from the apex (top left) to the diaphragm (bottom right) in zero end-expiratory pressure conditions. The entire lung is composed of nonaerated (white colour) or poorly aerated lung regions (grey colour) and there is no normally aerated lung region (black colour). The functional residual capacity is $265 \mathrm{~mL}$, the lung tissue volume of upper lobes is $953 \mathrm{~mL}$ (normal values $400-550 \mathrm{~mL}$ ) and the lung tissue volume of lower lobes is $568 \mathrm{~mL}$ (normal values $350-520 \mathrm{~mL}$ ). A lower inflexion point is present on the pressure/volume curve at $12 \mathrm{cmH}_{2} \mathrm{O}$ and the slope is $50 \mathrm{~mL} \cdot \mathrm{cmH}_{2} \mathrm{O}^{-1}$. A positive end-expiratory pressure of $10 \mathrm{cmH}_{2} \mathrm{O}$ induced a lung recruitment of $614 \mathrm{~mL}$ without any detectable pulmonary overinflation. such patients with diffuse and bilateral CT attenuations, arterial hypoxaemia is severe and the mortality rate is $>70 \%$. A primary insult to the lung is the most frequent cause of ARDS. The typical radiological presentation is made of bilateral and diffuse hyperdensities resulting in "white lungs" [32]. In one third of patients with ARDS, the aeration of upper lobes is preserved despite a regional excess of lung tissue and the loss of aeration involves exclusively lower lobes (fig. 2). When the regional loss of aeration is caused by alveolar flooding, a marked increase in lung tissue is observed, the overall lower lobe volume is preserved and bilateral radiological densities of the lower quadrants are present erasing the diaphragmatic cupola. When the regional loss of aeration is caused by compression atelectasis, a moderate or no increase in lung tissue is observed, the overall lower lobe volume is markedly reduced and bilateral radiological densities of the lower quadrants are discrete leaving apparent the diaphragmatic cupola. In such patients with "focal" CT attenuations, arterial oxygenation impairment is severe contrasting with the lack of extensive radiological abnormalities and the mortality rate is $\sim 40 \%$ [37]. In the remaining patients with ARDS, the loss of aeration involves massively lower lobes and only partially upper lobes (fig. 3). In such patients with "patchy" CT attenuations, ARDS is frequently caused by a primary insult to the lung, the radiological presentation is made of bilateral and diffuse X-ray densities respecting lung

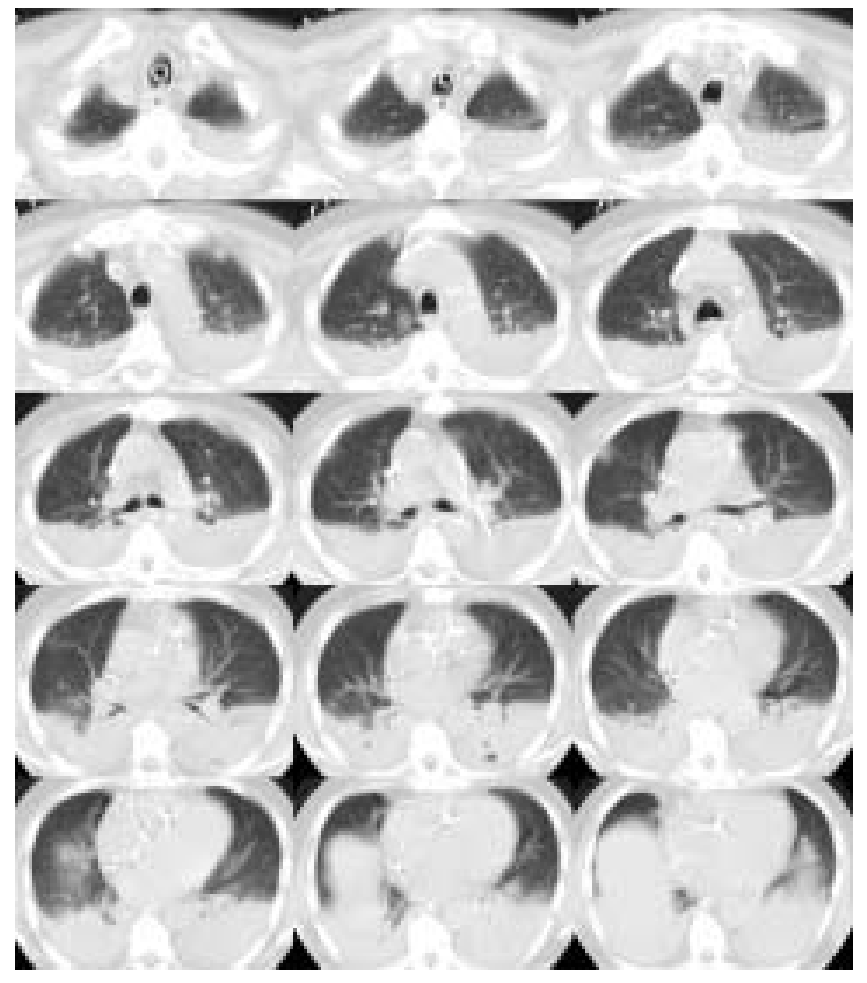

Fig. 2.-Computed tomography (CT) scan obtained in a 73-yr-old patient with "focal" acute respiratory distress syndrome caused by bronchopneumonia. Contiguous $1-\mathrm{cm}$ thick CT sections were obtained from the apex (top left) to the diaphragm (bottom right) in zero endexpiratory pressure conditions. The upper lobes appear normally aerated (black colour) despite a $40 \%$ increase in regional lung tissue, whereas lower lobes are either poorly (grey colour) or nonaerated (white colour). The functional residual capacity is $1004 \mathrm{~mL}$, the lung tissue volume of upper lobes $700 \mathrm{~mL}$ (normal values $400-550 \mathrm{~mL}$ ) and the lung tissue volume of lower lobes $515 \mathrm{~mL}$ (normal values $350-520 \mathrm{ml}$ ). On the pressure/volume curve, the lower inflexion point is absent and the slope is $53 \mathrm{~mL} \cdot \mathrm{cmH}_{2} \mathrm{O}^{-1}$. A positive end-expiratory pressure of $10 \mathrm{cmH}_{2} \mathrm{O}$ induced a lung recruitment of $200 \mathrm{ml}$ associated to a lung overinflation of $27 \mathrm{~mL}$. 


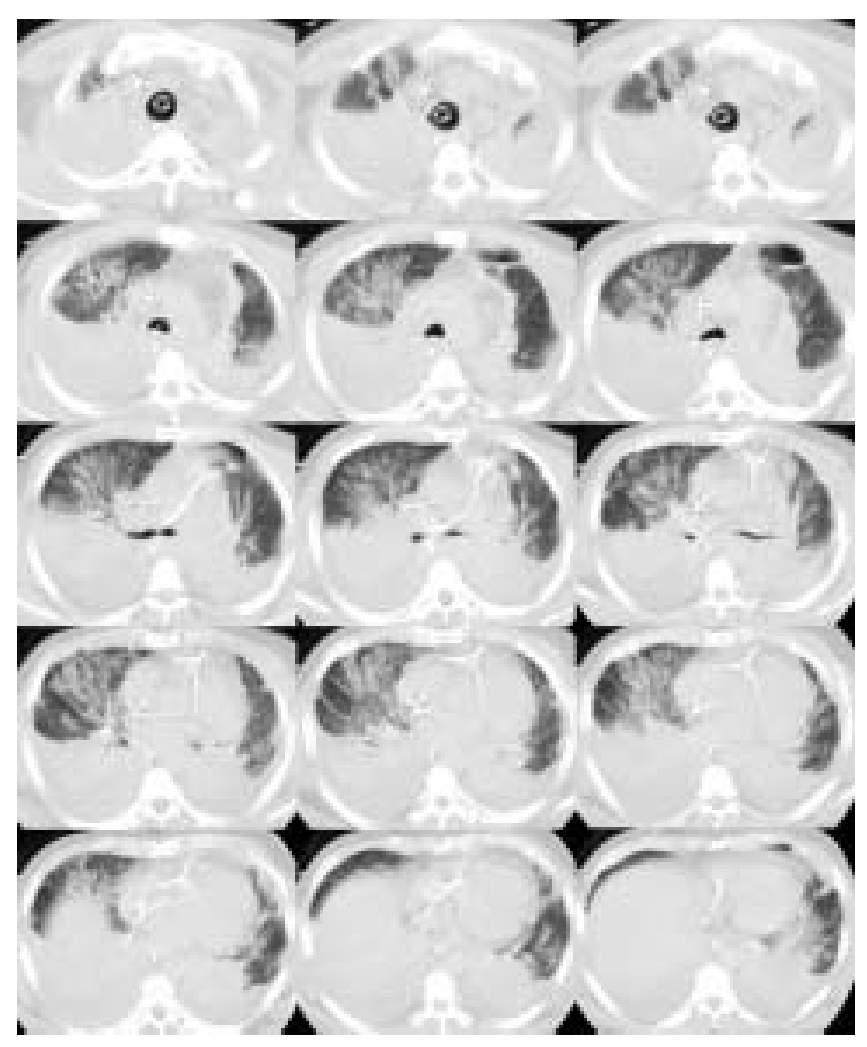

Fig. 3.-Computed tomography (CT) scan obtained in a 51-yr-old patient with "patchy" acute respiratory distress syndrome caused by aspiration. Contiguous $1 \mathrm{~cm}$-thick CT sections were obtained from the apex (top left) to the diaphragm (bottom right) in zero endexpiratory pressure conditions. Lower lobes are either poorly aerated (grey colour) or nonaerated (white colour) whereas some parts of the upper lobes remain normally aerated (black colour). The functional residual capacity is $472 \mathrm{~mL}$ and the lung tissue volume of upper lobes is $736 \mathrm{~mL}$ (normal values $400-550 \mathrm{~mL}$ ) and the lung tissue volume of lower lobes $895 \mathrm{~mL}$ (normal values 350-520 mL). On the pressure/ volume curve, the lower inflexion point is at $4 \mathrm{cmH}_{2} \mathrm{O}$ and the slope is $54 \mathrm{~mL} \cdot \mathrm{cmH}_{2} \mathrm{O}^{-1}$. A positive end-expiratory pressure of $10 \mathrm{cmH}_{2} \mathrm{O}$ induced a lung recruitment of $189 \mathrm{~mL}$ without detected alveolar overinflation.

apices, arterial hypoxaemia is severe and the mortality rate is $\sim 40 \%$.

In many patients lying in the supine position, atelectasis becomes predominant caudally to the diaphragmatic cupola [36]. In deeply sedated patients, the diaphragm behaves as a passive structure which moves upward in the rib cage [41] and transmits to lower lobes the increased abdominal pressure resulting from abdominal surgery and/or abdominal trauma. It has to be outlined that the lung injury itself can increase abdominal pressure. It is well known since the mid 1940s that abdominal pain and distension can be the revealing signs of an acute lung injury [44]. In fact, caudal and dependent parts of the lungs are not only compressed by the abdominal content [41] but also by the heart [45] and the accumulation of fluid in the pleural space. In the supine position, there is a nondependent to dependent decrease in regional aeration that is maximum in the juxta-diaphragmatic parts of the rib cage $[36,41]$. In lung regions located beneath the heart, the loss of aeration is massive and significantly greater than in lung regions located outside the ventricles' limits [45]. Despite the lack of left ventricular failure, the heart is enlarged and heavier in ARDS patients compared to healthy volunteers [45]. Myocardial oedema, hyperdynamic profile and pulmonary hypertension-induced right ventricular dilation are potential mechanisms that may contribute to the increased cardiac mass and dimensions in ARDS patients. Finally, in the supine position, lung tissue structures including pulmonary vessels are squeezed by different forces acting on caudal parts of the ribcage, a compression that could limit the plasma leakage through the injured alveolar-capillary barrier and explain why atelectasis becomes predominant beyond the diaphragmatic cupola.

In the 1990s, it was believed that the overall volume of the ARDS lung was preserved because the loss of gas was replaced by an excess of lung tissue [46]. This hypothesis based on CT data obtained from a single juxta-diaphragmatic CT section was not verified on the whole lung: multiple CT sections clearly demonstrated that the cephalocaudal dimensions of the ARDS lung are markedly reduced essentially at the expense of lower lobes [41]. In fact, the ARDS lung is made up of a combination of alveolar flooding, interstitial inflammation and atelectasis. In cephalic parts of the lung where external compressive forces are absent, alveolar flooding when present induces a massive loss of aeration. An illustrative example is provided by patients with diffuse CT attenuations: because the loss of gas is compensated by the excess of lung tissue, the upper lobes volume (gas + tissue) remains unchanged [32]. In contrast, in caudal parts of the lungs where external compressive forces (abdominal content, cardiac mass and pleural fluid effusion) are maximum in the supine position, atelectasis and alveolar flooding are frequently associated and the final reduction of overall lung volume and excess in lung tissue depend on the relative importance of each mechanism. This view of the ARDS lung has therapeutic consequences: re-establishment of a lung aeration providing adequate gas exchange at safe inspired fraction of oxygen not only requires an increase in intrathoracic pressure but also the relief of the external forces compressing lower lobes by adequate body positioning. Prone and semi-recumbent positions recruit dependent and caudal lung regions and improve gas exchange by partially relieving heart and abdominal compressions [47, 48].

\section{The lung morphology pattern determines the radiological presentation and the response to positive end-expiratory pressure}

Except in patients with diffuse ARDS whose radiographic and CT aspects are often concordant showing bilateral "white lungs", bedside frontal chest radiography is often misleading in patients with focal or patchy ARDS. In a series of 70 ARDS patients, bedside frontal chest radiography only correctly identified lung morphology in $41 \%$ of the patients, the highest rate of error being observed in patients with focal ARDS [37]. Surprisingly, in some severely hypoxaemic patients, frontal bedside chest radiography remains grossly normal and only a few basal hyperdensities can be identified. Only indirect signs such as the visualisation of the small fissure immediately above the right diaphragmatic cupola suggest a major reduction in the volume of lower lobes. This radiological presentation which often confuses the clinician, generally corresponds to nonaerated and partially atelectatic lower lobes which stand on the posterior face of the diaphragm and caudally to the diaphragmatic cupola. Despite the apparent preservation of lung aeration on the frontal chest radiography, the lung volume loss concerns at least $50 \%$ of the normal lung volume and explains the severe impairment of arterial oxygenation. In fact, the aeration present on frontal chest radiography concerns essentially upper lobes which, paradoxically, are characterised by a substantial increase in lung tissue. In such patients, lung CT appears as quite contributive for assessing lung morphology, quantifying the 
lung volume loss and understanding the causative mechanisms of arterial oxygenation impairment.

In a majority of patients with ARDS, a variable proportion of the lung parenchyma remains normally aerated at zero end-expiratory pressure [32] resulting in an uneven distribution of regional compliances [36]. The upper lobes appear more compliant than the lower lobes and any increase in intrathoracic pressure increases their distension before recruiting nonaerated pulmonary areas [36]. In such patients, applying PEEP $>10 \mathrm{cmH}_{2} \mathrm{O}$ results in overinflation of the aerated lung regions while concurrently, nonaerated areas begin to be recruited $[3,35,36,49]$. These results have been experimentally confirmed in dogs with oleic acid-injured lungs where regional lung volumes were measured using the parenchymal marker technique $[50,51]$. In the minority of patients whose ARDS is characterised by a diffuse and bilateral loss of aeration, the risk of overinflation appears more limited [3, 34, 36]. The lack of normally aerated lung regions at zero end-expiratory pressure explains why a PEEP as high as $17 \mathrm{cmH}_{2} \mathrm{O}$ does not induce any detectable lung overinflation [3]. The most severe forms of lung infection, pulmonary contusion, aspiration pneumonia fat embolism, amniotic embolism and near drowing are characterised by a diffuse loss of lung aeration whereas less severe forms of primary ARDS show a focal loss of aeration [36, 37]. Most secondary ARDS patients are also characterised by a focal loss of aeration [37]. As shown in a series of 69 patients with ARDS [36], primary and secondary ARDS patients do not differ as far as basal cardiorespiratory parameters, cardiorespiratory effects of PEEP and survival. The nature of lung injury (primary or secondary) does not influence the response to PEEP per se as previously suggested [52] but rather through its effects on lung morphology.

The experimental and clinical finding that lung overinflation and alveolar recruitment occur simultaneously in many patients or animals with ARDS forms the rationale for selecting the "right" PEEP level. The optimal PEEP for a given ARDS patient can be defined as the PEEP allowing an optimisation of arterial oxygenation without introducing a risk of oxygen toxicity and ventilator-induced lung injury [5]. In the majority of ARDS patients whose significant parts of the lungs remain normally aerated at zero end-expiratory pressure, a PEEP trial limited to $5-12 \mathrm{cmH}_{2} \mathrm{O}$ can be recommended in order to avoid overinflation of aerated lung regions that would inevitably result from the high intrathoracic pressures required to recruit some of the nonaerated pulmonary areas. In other words, the injured lung cannot be entirely reaerated without introducing a risk of ventilatorinduced lung injury. In the minority of patients without a single lung region normally aerated at zero end-expiratory pressure, a PEEP trial between $10-25 \mathrm{cmH}_{2} \mathrm{O}$ can be safely performed and the concept of keeping the lung fully aerated may be accepted [53]. In both situations, the use of periodic sighs could be useful [54-56]. CT has also provided evidence that end-expiratory aeration is markedly dependant on the preceding inspiratory plateau pressure: the higher the inspiratory plateau pressure, the more the PEEP prevents end-expiratory lung derecruitment [57].

\section{Mechanisms of the loss of aeration and their consequences on ventilator-induced lung injury}

The widely accepted hypothesis explaining the dependent loss of aeration is based on the "sponge" model developed by GATTINONI et al. [58] from CT scan studies performed on a single juxta-diaphragmatic CT section: the sternovertebral gradient of aeration resulting from the increased tissue mass causes the lung to collapse under its own weight in dependent regions [59]. This theory implies that pulmonary oedema remains purely interstitial because alveolar oedema cannot be associated to alveolar collapse, the alveolar gas being replaced by fluid. It also implies a decrease in the overall lung volume because, for obvious anatomical reasons, the increase in the interstitial volume cannot compensate entirely for the decrease in the alveolar volume resulting from lung collapse. Recently, HUBMAYR [60] raised the possibility that the ARDS lung is derecruited because it is filled with fluid, but not collapsed. In a canine oleic acid-induced ARDS model, MARTYNOWICZ and co-workers [50, 51] did not find any evidence of collapse of dependent lung units and were unable to demonstrate any opening and collapse of lung units with mechanical ventilation using the parenchymal marker technique. The authors logically concluded that the presence of liquid and foam in alveoli and conducting airways was the mechanism causing the loss of lung aeration [61]. Paradoxically the distribution of "opening" and "closing" pressures of the injured lung has been precisely described in a canine oleic acid-induced ARDS model [57].

CT data of the whole lung obtained in patients with ARDS are also difficult to reconcile with the opening and collapse theory. According to the "sponge" model, the loss of overall lung volume should be equally distributed between upper and lower lobes in patients lying supine: the lung tissue in excess being homogeneously distributed within the injured lung, the excess lung weight should be observed equally in cephalic and caudal parts of the lung resulting in an equivalent reduction of regional lung volume. Although a marked reduction of the end-expiratory volume of lower lobes is effectively observed in patients with ARDS, the volume of upper lobes is not reduced $[32,41]$. In some patients, the end-expiratory volume of upper lobes is even increased when compared to healthy controls [32] as experimentally observed in oleic acid-injured lungs. In ARDS patients with diffuse CT attenuations, aeration loss represents $67 \%$ of the normal gas volume of upper lobes whereas there is a $132 \%$ increase in lobar tissue volume; as a consequence, the overall regional lung volume remains unchanged (fig. 4). In ARDS patients with "focal" CT attenuations, lung aeration of upper lobes remains normal despite a $85 \%$ increase in lung tissue, resulting in a $25 \%$ increase in overall regional lung volume, a finding that contradicts the hypothesis that interstitial oedema causes alveolar collapse in dependent lung regions [32]. These data clearly suggest that alveolar flooding rather than lung collapse is the primary mechanism for lung derecruitment in upper lobes. In contrast, partial lung collapse is always present in lower lobes and likely results from an external compression by the abdominal compartment (fig. 5) and the heart [45]. It seems hazardous to incriminate the excessive lung weight as a causative factor of the loss of aeration in lower lobes since substantial increases in lung tissue are not associated with lung collapse in upper lobes. In fact, the sternovertebral gradient of aeration likely results from the anatomical superposition of upper and lower lobes in the supine position, the cephalocaudal gradient of aeration being the most important determinant causing the partial collapse of lower lobes.

As hypothesised by HuBMAYR [60], ventilator-induced lung injury should be predominantly caused by overinflation of aerated lung regions if lung recruitment results from the displacement of the air-fluid interface from distal airways to the alveolar space. If the reopening of collapsed alveoli is the mechanism of lung recruitment, then ventilator-induced lung injury should predominantly concern distal bronchioles in dependent lung areas. Human data on ventilator-induced lung injury have reported both overinflation in aerated lung areas and bronchial distension in nonaerated lung regions [62]. Recently, these lesions were reproduced in ventilated piglets with Escherichia coli pneumonia, a model of focal 

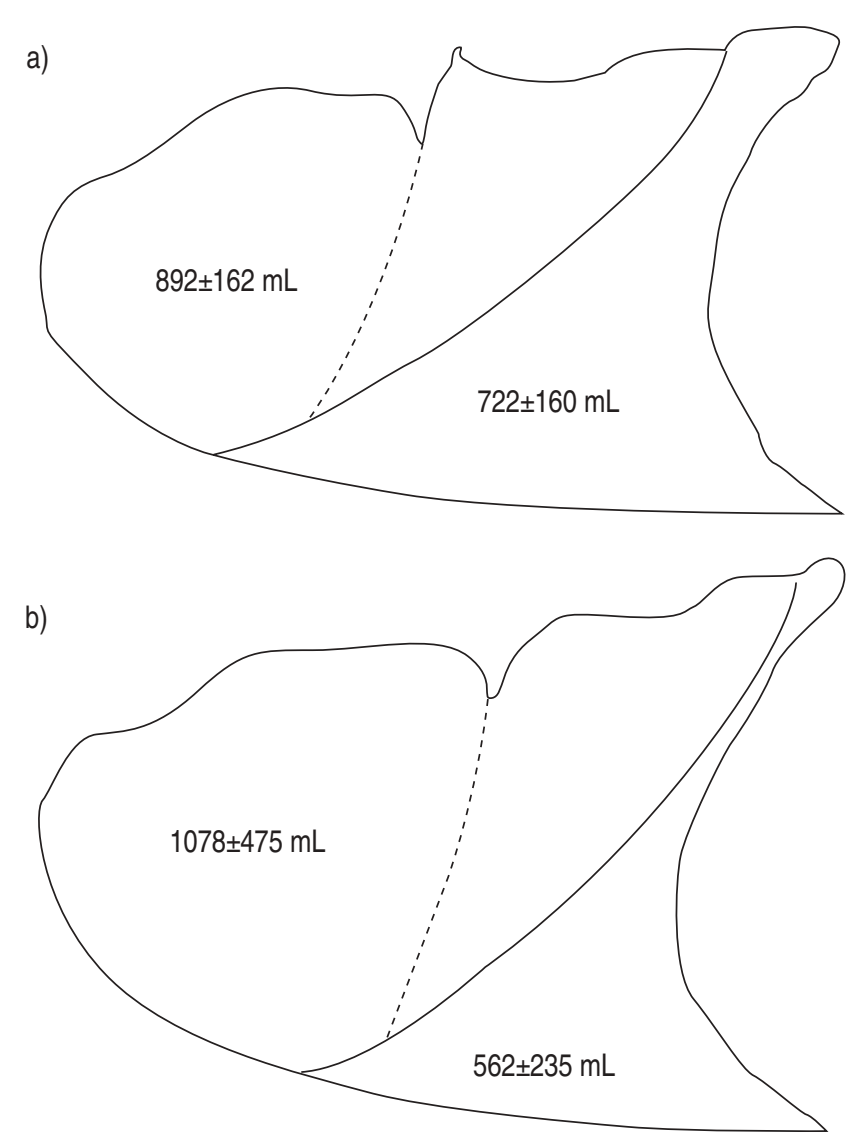

Fig. 4.-Comparative distribution of end-expiratory regional volumes of lung tissue, lung gas and overall lung volume (gas+tissue, see numbers within the figure) in a) healthy volunteers ( $\mathrm{n}=11$; upper lobes (gas: $642 \pm 135 \mathrm{~mL}$; tissue: $248 \pm 74 \mathrm{~mL}$ ); lower lobes (gas: $470 \pm$ $135 \mathrm{~mL}$; tissue: $251 \pm 88 \mathrm{~mL}$ )) and b) patients with acute respiratory distress syndrome $(\mathrm{n}=41$; upper lobes (gas: $434 \pm 249 \mathrm{~mL}$; tissue: $599 \pm 269 \mathrm{~mL}$ ); lower lobes (gas: $91 \pm 80 \mathrm{~mL}$; tissue: $498 \pm 167 \mathrm{~mL}$ )) lying in the supine position. The profile of the right lung is represented in the supine position with the small and main fissures represented by dashed and continuous lines respectively.

ARDS where alveoli are fluid filled and not collapsed [63]. Such results suggest that both mechanisms (partial reversal of alveolar flooding in upper and lower lobes and partial reopening of collapsed lung areas in lower lobes) are likely involved in mechanical ventilation-induced lung recruitment. However, very likely, lung collapse of caudal parts of the lung in the supine position does not result from the increased lung weight but from the external compression of lower lobes by the heart and the abdomen. This external compression explains why pressures much greater than $15 \mathrm{cmH}_{2} \mathrm{O}$ (the average anteroposterior lung dimension) are required to fully re-aerate the ARDS patients' lower lobes.

\section{Relationships between lung morphology and respiratory pressure/volume curves}

In ARDS characterised by a diffuse loss of aeration, the $P / V$ curve is essentially a lung recruitment curve and does not reflect lung mechanics of the "baby" lung [2, 57]. At zero end-expiratory pressure, all lung regions are poorly or nonaerated and each $\mathrm{mL}$ of gas penetrating within the respiratory system during the inflation procedure contributes to alveolar recruitment either by improving the aeration of poorly aerated lung regions and/or by re-inflating the nonaerated lung. In other words, the lung recruitment

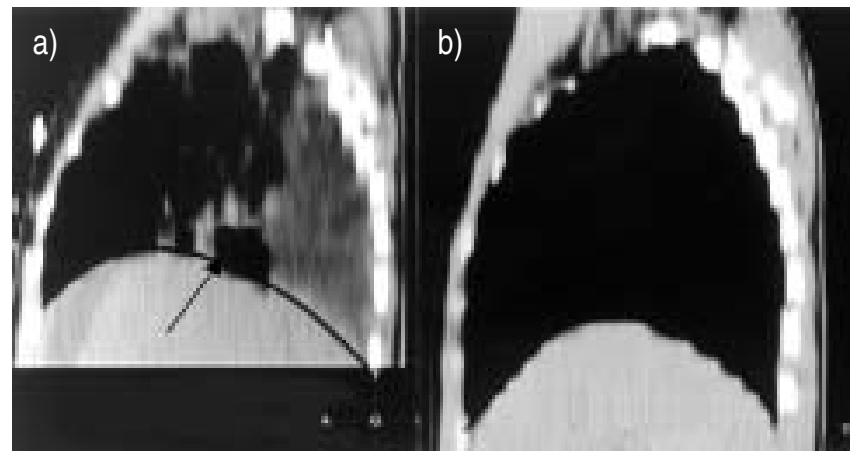

Fig. 5.-Comparative cephalocaudal dimensions measured from sagittal reconstruction of the right lung obtained from contiguous computed tomography sections in a) one patient with acute lung injury and b) one healthy volunteer. The arrow in a) indicates the direction of the pressure that the abdominal content exerts on the lung in a patient lying supine.

resulting from PEEP is equal to the increase in functional residual capacity induced by PEEP. The lower inflection point which is usually well defined corresponds to the pressure above which alveolar recruitment increases linearly with airway pressure. The initial part of the $P / V$ curve, named the starting compliance, is generally flat indicating that a minimum pressure is required to re-aerate the injured lung. The first $\mathrm{mL}$ of gas delivered to the respiratory system penetrate primarily into poorly aerated lung regions. Very likely, these lung regions are characterised by a gas-liquid interface within the alveolar space which alter regional lung mechanics and explains the low starting compliance. Then, with the progressive re-aeration of nonaerated lung regions, lung recruitment commences to be substantial and the chord compliance (the slope of the $P / V$ curve in its linear portion) becomes higher than the starting compliance. Experimentally, the lower inflection point can be caused either by the reopening of collapsed lung areas or by the re-inflation of an edematous lung in which all units are open $[61,64,65]$. By itself the presence of a prominent lower inflection point is not an indication of the mechanisms of lung aeration loss. The upper inflection point indicates the end of alveolar recruitment and the pressure above which alveolar overinflation commences. The slope of the $P / V$ curve determines the potential for recruitment. At the early stage of ARDS, the potential for recruitment is important in patients with diffuse loss of aeration [36]. As expected, the slope of the $P / V$ curve decreases with PEEP, attesting that the lung is progressively recruited [21,55]. Interestingly, $90 \%$ of ARDS characterised by a diffuse loss of aeration is caused by a direct insult to the lung and the hypothesis raised of a marginal recruitment in primary ARDS [52] has not been substantiated by further studies [21, 34, 36, 66].

In ARDS caused by a focal loss of aeration, the interpretation of the $P / V$ curve is much more complex. Lung recruitment of nonaerated lung regions as well as the mechanical properties of lung regions normally aerated at zero end-expiratory pressure contribute to the shape of the $P / V$ curve. The lower and upper inflection points are either absent or little prominent. The initial part of the $P / V$ curve, the starting compliance, is usually steep indicating that lung volume immediately increases at low pressures. Very likely the normally aerated lung is inflated and distended long before the recruitment of nonaerated lung regions commences. In the linear part of the $P / V$ curves, distension and recruitment occur simultaneously in different parts of the lung. At high pressure, overinflation of the aerated lung may appear whereas lung recruitment of nonaerated lung regions continues. These two 
a)

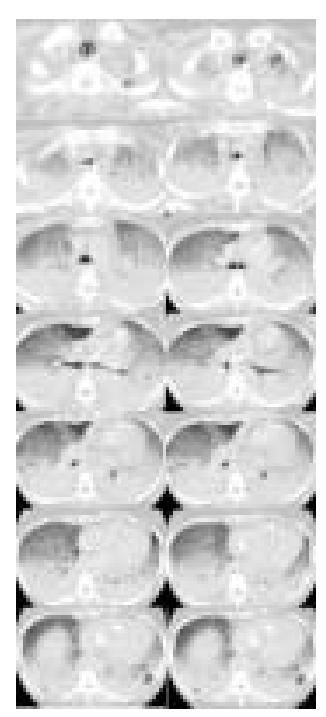

c)

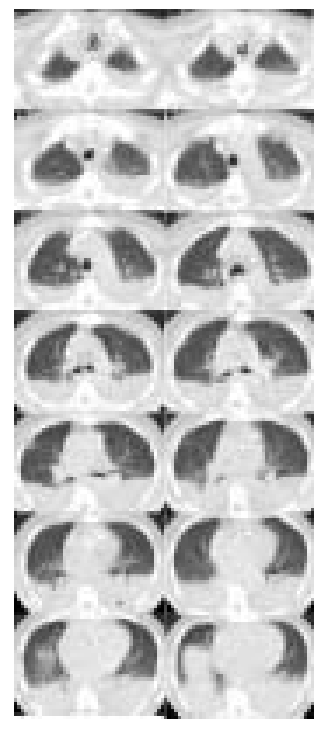

b)

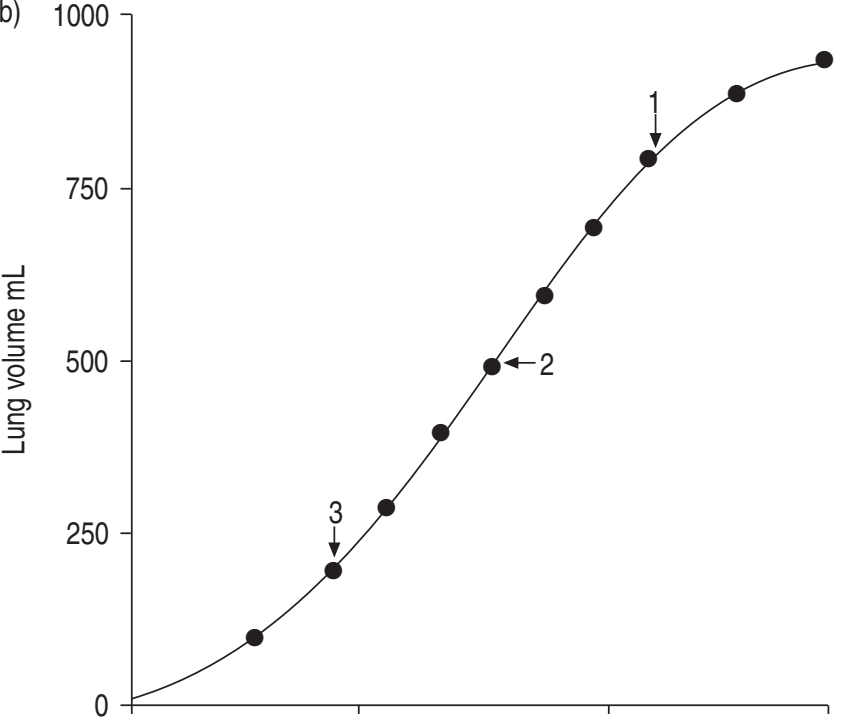

d)

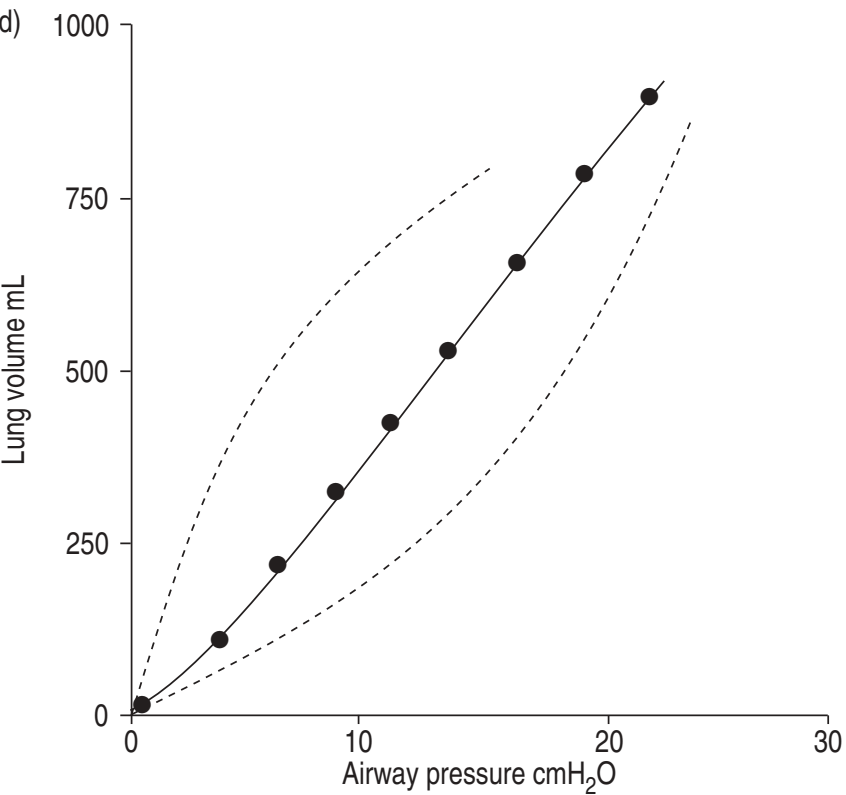

Fig. 6. - a) Computed tomography $(\mathrm{CT})$ scan and b) a respiratory pressure/volume $(P / V)$ curve obtained at zero end-expiratory pressure (ZEEP) in a patient with acute respiratory distress syndrome (ARDS) characterised by a diffuse loss of aeration. The $P / V$ curve is a lung recruitment curve since no part of the lung is normally aerated at ZEEP (1: upper inflection point=end of recruitment; 2: slope of curve=potential for recruitment; 3: lower inflection point=pressure at which the recruitment becomes linear). c) CT scan and d) a $P / V$ curve obtained at ZEEP in a patient with ARDS characterised by a focal loss of aeration. The $P / V$ curve is influenced by the respiratory mechanics of the normally aerated parts of the lung (upper dashed line) at ZEEP (on the CT scan shown in c) normally aerated parts of the lung are indicated in dark grey) and by the recruitment curve of poorly and nonaerated parts of the lungs (lower dashed line) at ZEEP (on the CT scan shown in c), poorly and nonaerated parts of the lung are indicated in light grey and white).

opposite effects explain why the upper inflection point is either absent or very progressive in many of these patients. A similar reasoning can be applied to the lower inflection point that is most often absent or progressive [32]. During the initial insufflation, normally aerated lung regions are the first to be inflated at low pressures long before the nonaerated lung is recruited. As a consequence, the lower inflection point that should correspond to this recruitment is blended into the progressive distension of the aerated lung. In ARDS patients with focal loss of aeration, keeping the plateau inspiratory pressure lower than the upper inflection point does not protect against lung overinflation. Similarly, the slope of the $P / V$ curve reflects not only the potential for recruitment but also the elastance of the aerated lung (fig. 6).

\section{Conclusion}

Computed tomography data obtained on the whole lung in patients with acute respiratory distress syndrome allow an understanding of the complex relationships existing between pressure/volume curves and lung morphology. 1) In normal healthy volunteers, the pressure/volume curve explores the mechanical properties of the respiratory system (lung+chest wall). 2) In acute respiratory distress syndrome patients in whom the loss of aeration is massive and diffuse at zero end-expiratory pressure, the pressure/volume curve is a lung recruitment curve and its slope indicates the potential for recruitment. 3) In acute respiratory distress syndrome patients in whom the loss of aeration is focally distributed, the 
pressure/volume curve is more complex to interpret because it is influenced by the mechanical properties of the lung remaining normally aerated at zero end-expiratory pressure and by the recruitment of the nonaerated lung. In these patients, who are the majority of acute respiratory distress syndrome patients, keeping the plateau inspiratory pressure below the upper inflection point is not an absolute protection against lung overinflation. As a consequence, selecting the adequate level of positive end-expiratory pressure in acute respiratory distress syndrome patients with a focal loss of aeration cannot be entirely based on the shape of the pressure/ volume curve. Because there is a risk of simultaneously inducing recruitment and overinflation in different parts of the lung with high intrathoracic pressures, positive endexpiratory pressure should be kept at a moderate level in acute respiratory distress syndrome patients with focal loss of aeration.

\section{References}

1. Gattinoni L, Pesenti A, Avalli L, Rossi F, Bombino M. Pressure-volume curve of total respiratory system in acute respiratory failure. Computed tomographic scan study. Am Rev Resp Dis 1987; 136: 730-736.

2. Hickling KG. The pressure-volume curve is greatly modified by recruitment. A mathematical model of ARDS lungs. Am J Respir Crit Care Med 1998; 158: 194-202.

3. Vieira SR, Puybasset L, Lu Q, et al. A scanographic assessment of pulmonary morphology in acute lung injury. Significance of the lower inflection point detected on the lung pressure-volume curve. Am J Respir Crit Care Med 1999; 159: 1612-1623.

4. Matamis D, Lemaire F, Harf A. Total respiratory pressurevolume curves in the adult respiratory distress syndrome. Chest 1984; 86: 58-66.

5. Rouby JJ, Lu Q, Goldstein I. Selecting the right level of positive end-expiratory pressure in patients with acute respiratory distress syndrome. Am J Respir Crit Care Med 2002; 165: 1182-1186.

6. Dall'Ava-Santucci J, Armaganidis A, Brunet F. Causes of error of respiratory pressure-volume curves in paralyzed subjects. J Appl Physiol 1988; 64: 42-49.

7. Gattinoni L, Mascheroni D, Bascilico E. Volume/pressure curve of total respiratory system in paralyzed patients: artifacts and correction factors. Intensive Care Med 1987; 13: $19-25$.

8. Sydow M, Burchardi H, Zinserling J. Improved determinated of static compliance by automated single volume steps in ventilated patients. Intensive Care Med 1991; 17: 108-114.

9. Levy P, Similowski T, Corbeil C. A method for studying the static volume-pressure curves of the respiratory system during mechanical ventilation. J Crit Care 1989; 4: 83-89.

10. Roupie E, Dambrosio M, Servillo G. Titration of tidal volume and induced hypercapnia in acute respiratory distress syndrome. Am J Respir Crit Care Med 1995; 152: 121-128.

11. Amato MBP, Barbas CSV, Mediros DM. Effect of a protective-ventilation strategy on mortality in the acute respiratory distress syndrome. N Engl J Med 1998; 338: 347354.

12. Ranieri VM, Mascia LM, Fiore T. Volume-pressure curve of the respiratory system predicts effects of PEEP in ARDS: "occlusion" versus "constant flow" technique. Am J Respir Crit Care Med 1994; 149: 19-27.

13. Ranieri VM, Mascia LM, Fiore T. Cardiorespiratory effects of positive end-expiratory pressure during progressive tidal volume reduction (permissive hypercapnia) in patients with acute respiratory distress syndrome. Anesthesiology 1995; 83: $710-720$

14. Servillo G, Svantesson C, Beyton L. Pressure-volume curves in acute respiratory failure. Automated low flow inflation versus occlusion. Am J Respir Crit Care Med 1997; 155: 1629-1636.

15. Lu Q, Vieira S, Richecoeur J. A simple automated method for measuring pressure-volume curve during mechanical ventilation. Am J Respir Crit Care Med 1999; 159: 275-282.

16. Suratt PM, Owens DH, Kilgore WT, Harry RR, Hsiao HS. A pulse method of measuring respiratory system compliance. J Appl Physiol 1980; 49: 1116-1121.

17. Suratt PM, Owens DH. A pulse method of measuring respiratory system compliance in ventilated patients. Chest 1981; 80: 34-38.

18. Mankikian B, Lemaire F, Benito S. A new device for measurement of pulmonary pressure-volume curves in patients on mechanical ventilation. Crit Care Med 1983; 11: 897-901.

19. Rodriguez L, Marquer B, Mardrus P. A new simple method to perform pressure-volume curves obtained under quasistatic conditions during mechanical ventilation. Intens Care Med 1999; 25: 173-179.

20. Gama AM, Meyer EC, Gaudencio AM, et al. Different low constant flows can equally determine the lower inflection point in acute respiratory distress syndrome patients. Artif Organs 2001; 25: 882-889.

21. Jonson B, Richard JC, Straus C, Mancebo J, Lemaire F, Brochard L. Pressure-volume curves and compliance in acute lung injury: evidence of recruitment above the lower inflection point. Am J Respir Crit Care Med 1999; 159: 11721178.

22. Karason S, Sondergaard S, Lundin S, Wiklund J, Stenqvist O. A new method for non-invasive, manoeuvre-free determination of "static" pressure-volume curves during dynamicl therapeutic mechanical ventilation. Acta Anaesthesiol Scand 2000; 44: 578-585.

23. Karason S, Sondergaard S, Lundin S, Wiklund J, Stenqvist O. Evaluation of pressure/volume loops based on intratracheal pressure measurements during dynamic conditions. Acta Anaesthesiol Scand 2000; 44: 571-577.

24. Karason S, Sondergaard S, Lundin S, Wiklund J, Stenqvist O. Direct tracheal airway pressure measurements are essential for safe and accurate dynamic monitoring of respiratory mechanics. A laboratory study. Acta Anaesthesiol Scand 2001; 45: 173-179.

25. Karason S, Sondergaard S, Lundin S, Stenqvist O Continuous on-line measurements of respiratory system, lung and chest wall mechanics during mechanic ventilation. Intensive Care Med 2001; 27: 1328-1339.

26. Ranieri VM, Brienza N, Santostasi S, et al. Impairment of lung and chest wall mechanics in patients with acute respiratory distress syndrome: role of abdominal distension. Am J Respir Crit Care Med 1997; 156: 1082-1091.

27. Mergoni M, Martelli A, Volpi A, Primavera S, Zuccoli P, Rossi A. Impact of positive end-expiratory pressure on chest wall and lung pressure-volume curve in acute respiratory failure. Am J Respir Crit Care Med 1997; 156: 846-854.

28. Karason S, Karlsen KL, Lundin S, Stenqvist O. A simplified method for separate measurements of lung and chest wall mechanics in ventilator-treated patients. Acta Anaesthesiol Scand 1999; 43: 308-315.

29. Baydur A, Behrakis PK, Zin WA, Jaeger M, Milic-Emili J. A simple method for assessing the validity of the esophageal balloon technique. Am Rev Respir Dis 1982; 126: 788-791.

30. Mull RT. Mass estimates by computed tomography: Physical density from CT numbers. Am J Radiol 1984; 143: 1101-1104.

31. Gattinoni L, Pesenti A, Torresin A, et al. Adult respiratoy distress syndrome profiles by computed tomography. J Thorac Imag 1986; 1: 25-30.

32. Puybasset L, Cluzel P, Gusman P, Grenier P, Preteux F, Rouby JJ. Regional distribution of gas and tissue in acute respiratory distress syndrome. I. Consequences for lung morphology. CT Scan ARDS Study Group. Intensive Care Med 2000; 26: 857-869. 
33. Malbouisson LM, Preteux F, Puybasset L, Grenier P, Coriat P, Rouby JJ. Validation of a software designed for computed tomographic (CT) measurement of lung water. Intensive Care Med 2001; 27: 602-608.

34. Malbouisson LM, Muller JC, Constantin JM, Lu Q, Puybasset L, Rouby JJ. Computed tomography assessment of positive end-expiratory pressure-induced alveolar recruitment in patients with acute respiratory distress syndrome. Am J Respir Crit Care Med 2001; 163: 1444-1450.

35. Vieira S, Puybasset L, Richecoeur J, et al. A lung computed tomographic assessment of positive end-expiratory pressureinduced lung overdistension. Am J Resp Crit Care Med 1998; 158: 1571-1577.

36. Puybasset L, Gusman P, Muller J-C, et al. Regional distribution of gas and tissue in acute respiratory distress syndrome - part 3: Consequences for the effects of positive end expiratory pressure. Intensive Care Med 2000; 26: 12151227.

37. Rouby JJ, Puybasset L, Cluzel P, Richecoeur J, Lu Q, Grenier P. Regional distribution of gas and tissue in acute respiratory distress syndrome. II. Physiological correlations and definition of an ARDS Severity Score. CT Scan ARDS Study Group. Intensive Care Med 2000; 26: 1046-1056.

38. Gattinoni L, D'Andrea L, Pelosi P, Vitale G, Pesenti A, Fumagalli R. Regional effects and mechanism of positive end-expiratory pressure in early adult respiratory distress syndrome. JAMA 1993; 269: 2122-2127.

39. Gattinoni L, Pesenti A, Bombino M, et al. Relationships between lung computed tomographic density, gas exchange, and PEEP in acute respiratory failure. Anesthesiology 1988; 69: 824-832.

40. Lu Q, Malbouisson LM, Mourgeon E, Goldstein I, Coriat P, Rouby JJ. Assessment of PEEP-induced reopening of collapsed lung regions in acute lung injury: are one or three CT sections representative of the entire lung? Intensive Care Med 2001; 27: 1504-1510.

41. Puybasset L, Cluzel P, Chao N, et al. A computed tomography assessment of regional lung volume in acute lung injury. Am J Resp Crit Care Med 1998; 158: 1644-1655.

42. Gevenois PA, Vuyst P, Maertelaer V, et al. Comparison of computed density and microscopic morphometry in pulmonary emphysema. Am J Respir Crit Care Med 1996; 154: 187-192.

43. Gould GA, Macnee W, Mclean A, et al. CT measurements of lung density in life can quantitate distal airspace enlargement - an essential defining feature of human emphysema. Am Rev Respir Dis 1988; 137: 380-392.

44. Mondor H. Diagnostics urgents abdomen. Masson Editeur. Paris, Librairies de L'Académie de Médecine de Paris, 1940; pp. 36-39.

45. Malbouisson LM, Busch CJ, Puybasset L, Lu Q, Cluzel P, Rouby JJ. Role of the heart in the loss of aeration characterizing lower lobes in acute respiratory distress syndrome. CT Scan ARDS Study Group. Am J Respir Crit Care Med 2000; 161: 2005-2012.

46. Gattinoni L, Pelosi P, Pesenti A, et al. CT scan in ARDS; clinical and physiopathological insights. Acta Anaesthesiol Scand 1991; 35: Suppl. 95, 87-96.

47. Gattinoni L, Pelosi P, Vitale G, Pesenti A, D'andrea L, Mascheroni D. Body position changes redistribute lung computed tomographic density in patients with acute respiratory failure. Anesthesiology 1991; 74: 15-23.

48. Richard JC, Maggiore SM, Michard F, et al. Upright positioning (UP) in patients with Acute Lung Injury (ALI). Am J Respir Crit Care Med 1999; 159: A695.
49. Dambrosio M, Roupie E, Mollet JJ, et al. Effects of positive end-expiratory pressure and different tidal volumes on alveolar recruitment and hyperinflation. Anesthesiology 1997; 87: 495-503.

50. Martynowicz MA, Walters BJ, Hubmayr RD. Mechanisms of recruitment in oleic acid-injured lungs. $J$ Appl Physiol 2001; 90: 1744-1753.

51. Martynowicz MA, Minor TA, Walters BJ, Hubmayr RD. Regional expansion of oleic acid-injured lungs. Am J Respir Crit Care Med 1999; 160: 250-258.

52. Gattinoni L, Pelosi P, Suter PM, Pedoto A, Vercesi P, Lissoni A. Acute respiratory distress syndrome caused by pulmonary and extrapulmonary disease. Different syndromes? Am J Respir Crit Care Med 1998; 158: 3-11.

53. Lachmann B. Open up the lung and keep the lung open. Intensive Care Med 1992; 18: 319-321.

54. Pelosi P, Cadringher P, Bottino N, et al. Sigh in acute respiratory distress syndrome. Am J Respir Crit Care Med 1999; 159: 872-880.

55. Richard JC, Maggiore SM, Jonson B, Mancebo J, Lemaire F, Brochard L. Influence of tidal volume on alveolar recruitment. Respective role of PEEP and a recruitment maneuver. Am J Respir Crit Care Med 2001; 163: 1609-1613.

56. Van Der Kloot TE, Blanch L, Youngblood AM, et al. Recruitment maneuvers in three experimentals models of acute lung injury. Effects on lung volume and gas exchange. Am J Respir Crit Care Med 2000; 161: 1485-1494.

57. Pelosi P, Goldner M, McKibben A, et al. Recruitment and derecruitment during acute respiratory failure: an experimental study. Am J Respir Crit Care Med 2001; 164: 122130.

58. Gattinoni L, Caironi P, Pelosi P, Goodman LR. What has computed tomography taught us about the acute respiratory distress syndrome? Am J Respir Crit Care Med 2001; 164: 1701-1711.

59. Pelosi P, D'andrea L, Pesenti A, Gattinoni L. Vertical gradient of regional lung inflation in adult respiratory distress syndrome. Am J Respir Crit Care Med 1994; 149: $8-13$.

60. Hubmayr RD. Perspective on lung injury and recruitment: a skeptical look at the opening and collapse story. Am J Respir Crit Care Med 2002; 165: 1647-1653.

61. Wilson TA, Anafi RC, Hubmayr RD. Mechanics of edematous lungs. J Appl Physiol 2001; 90: 2088-2093.

62. Rouby JJ, Lherm T, Martin de Lassale E, et al. Histologic aspects of pulmonary barotrauma in critically ill patients with acute respiratory failure. Intensive Care Med 1993; 19: 383-389.

63. Goldstein I, Bughalo MT, Marquette CH, Lenaour G, Lu Q, Rouby JJ. Mechanical ventilation-induced air-space enlargement during experimental pneumonia in piglets. Am J Respir Crit Care Med 2001; 163: 958-964.

64. Suki B, Andrade JS Jr, Coughlin MF, et al. Mathematical modeling of the first inflation of degassed lungs. Ann Biomed Eng 1998; 26: 608-617.

65. Martin-Lefevre L, Ricard JD, Roupie E, Dreyfuss D, Saumon G. Significance of the changes in the respiratory system pressure-volume curve during acute lung injury in rats. Am J Respir Crit Care Med 2001; 164: 627-632.

66. Rialp G, Betbese AJ, Perez-Marquez M, Mancebo J. Shortterm effects of inhaled nitric oxide and prone position in pulmonary and extrapulmonary acute respiratory distress syndrome. Am J Respir Crit Care Med 2001; 164: 243249. 